\title{
Metamaterials for MIMO
}

\author{
R. Seviour ${ }^{(1)}$ and A. Hopper ${ }^{(1)}$, \\ (1) University of Huddersfield, Huddersfield, UK
}

\begin{abstract}
Since their first realization the development of metamaterial based devices and applications has gained an increasing level of interest. These materials offer the opportunity to specifically engineer the materials ability to, control, enhance and suppress EM wave propagation though and near these materials, either in a specific direction or altogether. These materials have been used to construct a range of novel microwave devices such as antennas [1,2], phase-shifters [3], couplers [4], broadband compact power-dividers [5] and other devices such as beam steerers, modulators, band-pass filters and lenses.
\end{abstract}

More recently researchers have considered the use of metamaterials for MIMO application, either as antenna or parasitic/absorptive elements to control interference. In this paper we present an overview of metamaterials, looking at design, fabrication, and characterization. Focused towards the ability of metamaterials to offer a tailored EM mm-wave response for novel MIMO and 5G applications.

\section{Introduction}

The ability of engineers to develop new RF technologies is largely limited only by the EM properties of available materials. For over 100 years materials with specific RF properties, such as Polytetraflufoethylene (Teflon) and $\mathrm{HfO}_{2}$, have been artificial synthesised at the molecular level and used by Engineers, to modify EM wave propagation and interactions in devices. The interaction between EM wave and material is described via the constuitive relations, in a simplistic form are;

$$
\begin{aligned}
& D(k, \omega)=\varepsilon(k, \omega) E(k, \omega) \\
& B(k, \omega)=\mu(k, \omega) H(k, \omega)
\end{aligned}
$$

the permittivity $(\varepsilon)$ and the permeability $(\mu)$ are the averaged EM response functions of the molecules in the material to the electric component $(\varepsilon)$ and the magnetic component $(\mu)$. These response functions are averaged over all molecules in a volume $\sim \lambda^{3}$, an approximation that holds well into ultraviolet even for rare gases. Molecular synthesised EM materials have led to the creation of a range of novel RF technologies, although the parameter range these materials can access is limited. To expand this range researchers have developed artificial composite structures with, periodic, sub-wavelength inclusions. These inclusions appear, to the incident RF, identical to giant molecules with a large polarizability. Enabling the EM interaction with these collective inclusions to be described in terms of the "homogenised" abstracted bulk material parameters permittivity and permeability, treating the structure as an "effective media" or an "artificial material".

\section{Artificial Materials}

Research into artificial materials began in the 1890s, when Rayleigh considered an array of subwavelength small metallic spheres as a continuous medium[6]. The first applications of Artificial materials appeared in 1940s with the pioneering work of Kock who created Artificial Dielectrics from arrays of sub-wavelength metallic structures (spheres, rods, plates) to form Dielectric Lenses[7]. Although all these effective media either had a negative permittivity (permeability) and positive permeability (permittivity), or both permittivity and permeability were positive. The important point is that there are no naturally occurring materials that have a simultaneous negative permittivity and permeability. These double negative materials are referred to as metamaterials, a term first coined by Walser 1999, who defined a metamaterial as "...macroscopic composites having man-made, three-dimensional, periodic cellular architecture designed to produce an optimised combination, not available in nature, of two or more responses to specific excitation". The first systematic theoretical study of metamaterials is attributed to Veselago[8]. His theoretical study showed that for a monochromatic uniform plane wave in such a medium the direction of the Poynting vector is antiparallel to the direction of the phase velocity, as well as presenting the possibility of a lens constructed from this material.

\subsection{Artificial Atoms}

Pendry[9] presented the key sub-wavelength elements that could be used to construct the unit-cells (artificial atoms) of a metamaterial. The Split Ring Resonator (SRR) remains the meta-atom of choice for researchers, where the basic geometry remains the same as that originally proposed by Schelkunoff in 1950. Figure 1 (a) shows the double SRR designed by Pendry[9]. For a system of SRRs to be considered an "artificial materials" each unit-cell (individual SRR) must be less than $\lambda / 10$ in size, and have at least enough unit-cells to hold one wavelength. These 
size constraints ensure we meet the homogeneity condition necessary to treat our system as an effective media.
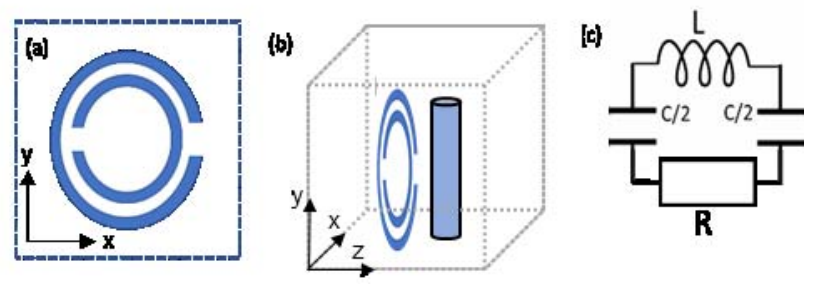

Figure 1. (a) double SRR geometry building block, and an array of SRRs. (b) combined SRR with wire array. (c) the equivalent circuit diagram from the SRR shown in (a).

Starting with just a "single ring" of the form shown in figure 1, without the "split" in the ring the interaction would be purely inductive (non-resonant), resulting in a weakly diamagnetic system (permeability $<1$ ). The "split" prevents the current circulating, causing charge to collect (a capacitance), forming an equivalent LCR resonant circuit, shown in figure 1(c). The behaviour of this system is dominated by the resonance from the outer SRR, where the inner SRR offers greater control over the meta-atom parameters. As the EM field induced by the outer, dominate, SRR in the inner SRR opposes the inducing field. Suppressing the electric dipole moment, allowing the magnetic comment to dominate creating by design permeabilites $>>1$.

The inductance is relatively easy to determine as $L \approx 2 \mu_{0} r$, whereas the capacitance is complicated by the additional capacitive effects from the gap that separates the two SRRs. Although the capacitance of the split rings can be approximated by $C \approx \pi \varepsilon 0 \mathrm{rt} / 2 \mathrm{~d}$, $\mathrm{t}$ is the combined width of the rings and $d$ the separation between the rings. From this we estimate the resonant frequency of the meta-atom as $\omega_{0}=\left(1 /\left(L+R / j \omega_{0}\right) C\right)^{1 / 2}$. From which we can determine the effective permeability of a material formed from a lattice of these individual sub-wavelength SRRs [10];

$$
\mu_{e f f}(\omega)=1+\frac{\pi^{2} r^{4} \mu_{0}}{V\left(\omega_{0}^{2} / \omega^{2}-1\right) L}
$$

Where $\mathrm{V}$ is the unitcell volume for our individual metaatom. The second element of our meta-atom, used to control the effective permittivity in the unit cell is a simple wire, shown combined with the SRR in figure $1(\mathrm{~b})$. The effective permittivity of the wire is given by $\varepsilon_{e f f}=1-\omega_{\mathrm{p}}{ }^{2} / \omega^{2}$, where $\omega_{\mathrm{p}}$ is the plasma frequency of electrons in the wire. If we assume a lossless wire, then the wire array can be modelled as an array of inductors, with inductance $L$. In this case the effective permittivity becomes;

$$
\varepsilon_{e f f}(\omega)=1-\frac{1}{d^{2} \omega^{2} \varepsilon_{0} L}
$$

This approach does not take into account electric coupling or the bianisotropic nature of the material. Although it does enable us, at least to 1st order, to gain useful insights into how changes in geometry will change the effective permeability of our artificial material, i.e. $\omega_{0} / \omega<1$ results in a negative permeability. This approach enables us to establish the broad dimensions of a geometry that will produce a response at the appropriate frequency required.

\section{Parameter Determination}

In this section we determine the effective permittivity and permeability from measurements of the scattering parameters (S-parameters). The approach used is a variant of the Nicolson-Ross-Weir NRW approached adapted by Smith [11] to account for possible negative responses in the real components of the permittivity and permeability. The NRW uses a closed form expression allowing the complex form of the permittivity and permeability to be determined directly from S-parameter measurements. In addition the NRW technique is relatively robust to experimental error. Assuming the material is a free-standing slab (thickness d) surrounded by a vacuum, with normal incident plane waves, then the S-parameters S21 and S11 are given by;

$$
\begin{gathered}
S_{21}=\left[\cos (n k d)-\frac{i}{2}\left(Z+\frac{1}{Z}\right) \sin (n k d)\right]^{-1} \\
S_{11}=-\frac{i}{2}\left(Z+\frac{1}{Z}\right) \sin (n k d) \cdot S_{21}
\end{gathered}
$$

$k=2 \pi / \lambda_{0}$ is the free space wave vector, $Z=Z^{\prime}+i Z^{\prime \prime}$ the impedance of the material, and $n=n^{\prime}+n^{\prime \prime}$ the refractive index. Inverting these equations we find,

$$
\begin{gathered}
Z= \pm\left[\frac{\left(1+S_{11}\right)^{2}-S_{21}^{2}}{\left(1-S_{11}\right)^{2}-S_{21}^{2}}\right]^{1 / 2} \\
n^{\prime}= \pm \frac{1}{k d} \mathfrak{R}\left[\cos ^{-1}\left(\frac{1-S_{11}^{2}+S_{21}^{2}}{2 S_{11}^{2}}\right)\right]+\frac{2 \pi m}{k d} \\
n^{\prime \prime}= \pm \frac{1}{k d} \mathfrak{J}\left[\cos ^{-1}\left(\frac{1-S_{11}^{2}+S_{21}^{2}}{2 S_{11}^{2}}\right)\right]
\end{gathered}
$$

Where the permittivity and permeability can be found directly, $\varepsilon_{\text {eff }}=n / Z$ and $\mu_{\text {eff }}=n Z$. The complication of course is in choosing the root and branch of the above equations. For a passive media the imaginary components of $\varepsilon_{e f f}, \mu_{e f f}$, $n$ and the real component $\mathrm{Z}$ must be positive (except at points of anti-resonances). The inverse cosine introduces some ambiguity into the imaginary component of $\mathrm{n}$. To constrain the solution we ensure that n' is continuous across the frequency range.

\section{Metamaterial Sheet}

To investigate wave transport we model an infinite sheet of metamaterial using HFSS, the commercial finite element method solver for electromagnetic structures from Ansys. This is achieved by modelling the unit cell geometry shown in figure 2, using Bloch-Floquet boundary conditions to create the infinite sheet. To demonstrate the bi-isotropic behaviour of SRR based materials we consider wave 
propagation in two planes relative to the unit cell geometry, perpendicular figure 2(a) and parallel figure 2(b). The unit cell consists of a double $\mathrm{Cu}$ square SRR on an FR4 substrate with $\mathrm{Cu}$ wire strip on the back. Total unit cell size $3 \mathrm{~mm}$, the $\mathrm{Cu}$ track of the SRR has a $200 \mu \mathrm{m}$ width and is $2 \mathrm{~mm}$ long.

(a)

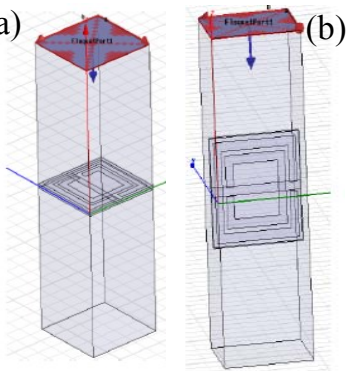

Figure 2. Meta-atom unit cell, wave propagation from the top (blue arrow) to the bottom Bloch-Floquet boundary conditions on all other sides. (a) propagation perpendicular to SRR. (b) propagation parallel to SRR.

The simulation was conducted in the frequency domain to determine the S-parameters both parallel and perpendicular to the structure. Using equations 5 and 6 to calculate the permittivity and permeability, shown in figures 3(a) and (b), for perpendicular and parallel wave propagation respectively. Figures 3(c) and (d) show the Absorption coefficient determined from the refractive index.
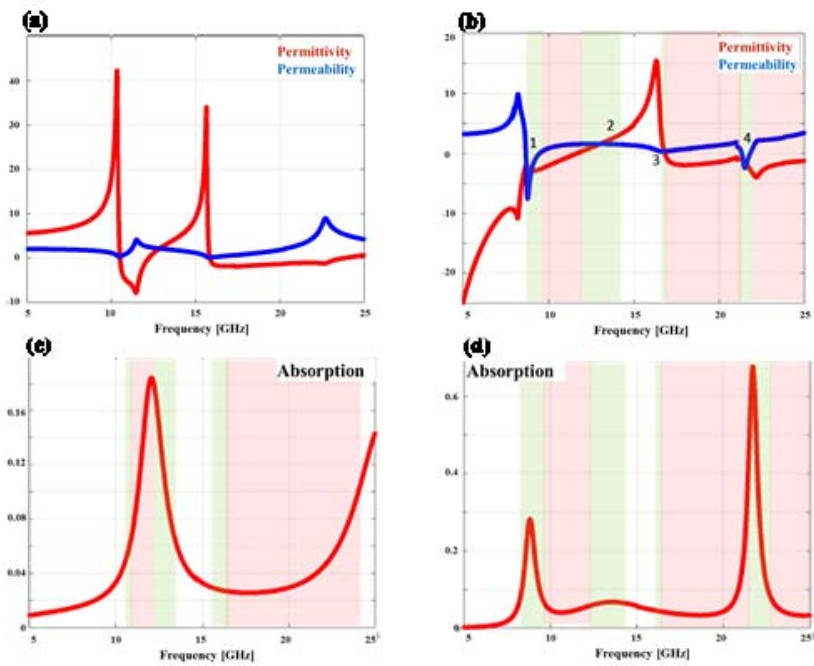

Figure 3. Permittivity and permeability (a) and (b), for perpendicular and parallel wave propagation respectively. (c) and (d) the Absorption coefficient.

From figure 3 we see the bi-isotropic nature of the metamaterial configuration, where regions of positive/negative permittivity and permeability are dependent on both direction of propagation and frequency. Likewise the Absorption shows a marked difference depending on direction, for example at $9 \mathrm{GHz}$ the Absorption coefficient is 0.03 for perpendicular transport, whereas for parallel transport the Absorption coefficient is
0.5. More interesting information can be gleamed from the dispersion relation for the material, which of course also displays the direction/frequency dependence, shown in figure 4. The dispersion behaviour has many interesting features, extreme slow wave velocity and negative dispersion. Note the cusps in figure 4 relate to high dispersive regions, the group velocity $\mathrm{V}_{\mathrm{g}} \approx \partial \omega / \partial k$ only holds true for non-dispersive media. A key aspect from figure 4 for different directions of propagation we notice band gaps where for specific frequencies wave propagation is not possible.
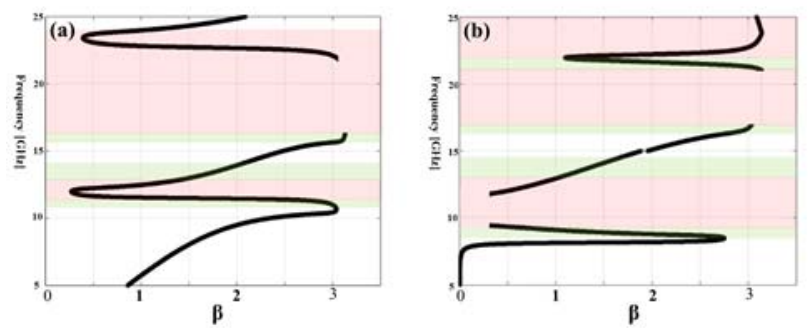

Figure 4. Dispersion relation for (a) perpendicular and (b) parallel wave propagation.

\section{MIMO}

A major advantage of using mm-waves around $60 \mathrm{GHz}$ is the Gigabit data rates that can be achieved. This is a prime driver in creating a growing interest in MIMO research at this frequency. Although working at $60 \mathrm{GHz}$ presents major challenges, in particular for MIMO systems where compactness and low-weight are a necessary feature in antenna design. This situation is exacerbated by the very short wavelengths $(\sim 5 \mathrm{~mm})$ at $60 \mathrm{GHz}$, which gives rise to several issues around the design and fabrication of the multiple antenna elements in MIMO arrays. As the antenna elements are generally placed between $\lambda / 8-\lambda / 2$ apart, at this frequency mutual coupling between antenna elements is a common problem in antenna. Where reducing this electromagnetic coupling between antenna elements is a challenging design task, and an active area of research.

Research into the suppression of mutual coupling between antennas has being an area of active research for around 40 years. For example, in [12] the use of a lossless network between input and the antenna ports is used, where the mutual coupling antenna impedance is purely reactive at resonance to isolate antennas from each other. Or using transmission lines as antenna decouplers [13,14]. Other approaches have used resonant defects and slits in the ground plane to confine resonate states $[15,16,17]$. As well as metamaterial and EM band-gap approaches [18].

All the above approaches focused on mutual coupling suppression at $3 \mathrm{GHz}$ and below, including the metamaterials approach (1-1.6 GHz). Attempting to scale these approaches to higher frequencies, especially $60 \mathrm{GHz}$ introduces its own issues. Although a correctly designed optimised metamaterial substrates for MIMO antennas can 
be used to control and mitigate the mutual coupling that arises between individual antenna elements even at $60 \mathrm{GHz}$ and above. As we have discussed metamaterials can be engineered with very specific properties, both frequency and direction of propagation specific. For example, we can use metamaterial constructs as a substrate for MIMO antennas designed to operate parasitically at the points of coupling, designed with spatially, directional, localised frequency band-gaps. We can even take the approaches used previously and design materials to implement these approaches at $60 \mathrm{GHz}$. For example using the type of structure presented in section 5, scaled to $60 \mathrm{GHz}$, orientated correctly EM should lead to a suppression of EM interaction across the material with an Absorption coefficient of 0.8 , inside a band-gap, in the plane between antenna elements, whilst perpendicular to the antenna elements the Absorption coefficient is $\sim 0.03$.

Fabrication at these frequencies can also be an issue, as to ensure homogeneity, so that our system can be treated as a material, each unit-cell must be less than $\lambda / 10$. Hence at 60 $\mathrm{GHz}$ we need our unit-cell to be smaller than $500 \mu \mathrm{m}$, with features around $50 \mu \mathrm{m}$. Fabrication of structures with this feature size can be fabricated with conventional lithographic processes. Currently these lithographic processes are restricted to the fabrication of 2dimensional objects. This does place a certain level of restriction on the range of structures that can be fabricated.

\section{References}

1. C. Caloz, T. Itoh, Microwave Symposium Digest, IEEE MTT-S International Microwave Symposium, vol. 1, June 2003, pp. 195-198.

2. F. Qureshi, M. Antoniades, G.V. Eleftheriades, IEEE Antenna Wireless Propagation Lett. 4 (2005) p. 333-336.

3. M. Antoniades, G.V. Eleftheriades, IEEE Antennas Wireless Propagation Lett. 2 (7) (2003) p. 103-106.

4. C. Caloz, A. Sanada, T. Itoh, IEEE Trans. Microwave Theory Techniques 52 (3) (2004) p. 980-992.

5. M. Antoniades, G.V. Eleftheriades, IEEE Microwave Guided Wave Lett. 15 (11) (2005) p. 808-810.

6. Rayleigh (1892) Phil. Mag, 5 (34), 481.

7. Kock, W.E. (1948) Bell System Technical J, 27, 58.

8. Veselago, V.G. (1967) Usp. Fiz. Nauk, 92, 517-526.

9. Pendry J.B., Holden A.J., D.R. and Stewart, W. (1999). IEEE Trans Microw Theory Tech, 47, 2075-2084.

10. Marques R., F.M. and Rafii-El-Idrissi, R. (2002). Phys $\operatorname{Rev} B, 65$ (144440).

11. D. R. Smith, D. C. Vier, T.K. and Soukoulis, C.M. (2005). Phys. Rev. E, 71 (036617).

12. J. Andersen and H. Rasmussen, IEEE Trans. Antennas Propag., vol. 24, no. 6, pp. 841-846, Nov. 1976.
13. S.-C. Chen, Y.-S. Wang, and S.-J. Chung, IEEE Trans. Antennas Propag., vol. 56, no. 12, pp. 3650-3658, Dec. 2008.

14. S. Dossche, S. Blanch, and J. Romeu, Proc. IEEE/ACES Int. Conf. on Wireless Communications and Applied Computational Electromagnetics, Apr. 2005, pp. 849-852.

15. C. Volmer, J. Weber, R. Stephan, K. Blau, and M. Hein, IEEE Trans. Antennas Propag., vol. 56, no. 2, pp. 360-370, Feb. 2008.

16. P. J. Ferrer, J. M. Gonzalez-Arbesu, and J. Romeu, Microw. Opt. Tech. Letts., vol. 50, no. 5, pp. 1414-1417, May 2008.

17. M. M. Bait-Suwailam, M. S. Boybay, and O. M. Ramahi, Proc. 13th Int. Symp. on Antenna Technology and Applied Electromagnetics (ANTEM/URSI), Feb. 2009, pp. $1-4$.

18. D. Sievenpiper, L. Zhang, R. Broas, N. Alexopolous, and E. Yablonovitch, IEEE Trans. Microw. Theory Tech., vol. 47, no. 11, pp. 2059-2074, Nov. 1999.

19. M. Bait-Suwailam, IEEE TRANS. Antennas and Propagation, 58, 9, 2010 